\title{
Z-alpha1-antitrypsin polymers and small airways disease: a new paradigm in alfa-1 anti- trypsin deficiency-related COPD development?
}

\author{
Laura Pini $^{1,2}$, Laura Tiberio ${ }^{3}$, Marianna Arici ${ }^{2}$, Luciano Corda ${ }^{1}$, Jordan Giordani ${ }^{2}$, Elena Bargagli ${ }^{4}$, \\ Claudio Tantucci ${ }^{1,2}$ \\ ${ }^{1}$ Respiratory Medicine Unit, ASST-Spedali Civili di Brescia; ${ }^{2}$ Department of Clinical and Experimental Sciences, \\ University of Brescia; ${ }^{3}$ Department of Molecular and Translational Medicine, University of Brescia; ${ }^{4}$ Respiratory \\ Diseases and Lung Transplantation, Department of Medical and Surgical Sciences and Neurosciences, Siena \\ University Hospital, Siena, Italy
}

\begin{abstract}
The presence of alpha1-antitrypsin (AAT) polymers, known to promote a sustained pro-inflammatory activity, has been pre-
\end{abstract}

Correspondence: Prof. Laura Pini, Department of Clinical and Experimental Sciences University of Brescia, Respiratory Medicine Unit, Spedali Civili di Brescia, Piazzale Spedali Civili 1, Brescia, Italy. Tel. +39.30.3996263.

E-mail: laura.pini@unibs.it

Key words: Alpha-1 anti-trypsin; polymers; small airways; AATD; AAT.

Ethics approval and consent to participate: The study was performed in accordance with the Helsinki declaration and was approved by the University Ethic Committee at the Department of Experimental and Clinical Science. All participants signed written informed consent upon enrolling.

Consent for publication: All participants of the study and the listed authors gave their consent for the publication of the manuscript.

Availability of data and material: All data generated or analyzed during this study are included in this published article.

Conflict of interest: The authors declare that they have no competing interests, and all authors confirm accuracy.

Contributions: LP, CT, study design; LT, MA, LC, data collection; LT, MA, data analysis; LP, LT, MA, LC, JG, EB, CT, interpretation of results, manuscript revision; LP, CT, manuscript initial drafting. All the authors have read and approved the final version of the manuscript and agreed to be accountable for all aspects of the work.

Received for publication: 10 April 2021.

Accepted for publication: 11 May 2021.

${ }^{\circ}$ Copyright: the Author(s), 2021

Licensee PAGEPress, Italy

Monaldi Archives for Chest Disease 2021; 91:1883

doi: 10.4081/monaldi.2021.1883

This article is distributed under the terms of the Creative Commons Attribution Noncommercial License (by-nc 4.0) which permits any noncommercial use, distribution, and reproduction in any medium, provided the original author(s) and source are credited. viously demonstrated in bronchial biopsies of subjects with ZAAT deficiency (AATD) suggesting a possible role in the development of COPD through a small airway disease impairment. The study aimed to assess the presence of small airways dysfunction and the potential correlation with the presence of ZAAT polymers obtained by Exhaled Breath Condensate (EBC) collection in PiZZ subjects, as compared with matched healthy PiMM subjects. We enrolled 19 asymptomatic, never smoker subjects: 9 PiZZ and 10 PiMM as controls, without obstructive ventilatory defect (i.e., normal $\mathrm{FEV} 1 / \mathrm{VC} \%$ ratio). All subjects underwent complete pulmonary function tests (PFT). EBC was collected in all subjects. ELISA test was applied to search for ZAAT polymers. The PiZZ subjects showed normal lung volumes and DLCO values. However, in comparison with PiMM subjects, the single breath test N2 wash-out revealed significant differences regarding the phase III slope $(1.45 \pm 0.35 \mathrm{~N} 2 / \mathrm{L} v s$ $0.96 \pm 0.40 \mathrm{~N} 2 / \mathrm{L})(\mathrm{p}<0.02)$ in the PiZZ subjects, while the closing volume/vital capacity ratio ( $14.3 \pm 4.5 \%$ vs $11.3 \pm 6.3 \%)$ was not significantly increased. The ELISA test detected the presence of Z-AAT polymers in $44 \%$ of $\mathrm{PiZZ}$ patients. Asymptomatic, never smoker PiZZ subjects with normal spirometry and lung diffusion capacity showed airways impairment when compared to PiMM subjects. Although Z-AAT polymers were found only in $44 \%$ of PiZZ subjects, these findings suggest the possibility that chronic bronchiolitis can develop as a result of the long-term pro-inflammatory activity of Z-AAT polymers in subjects with Z-related AATD.

\section{Introduction}

Alpha1-Antitrypsin deficiency (AATD) is one of the most common genetic disorders worldwide [1]. It is due to a genetic alteration on the gene Serpine1 on chromosome 14, resulting in a non- functional protein production [2-6]. Decreased levels of alpha1-antitrypsin (AAT) in the bloodstream are associated typically with panlobular emphysema [7] and liver disease, including hepatocellular carcinoma and liver cirrhosis $[8,9]$ but also with bronchial asthma and vascular diseases [10-13].

During the last 20 years, many studies focused on the pathogenetic pathways leading to lung and/or other diseases in AATD and particularly on the role of protein polymerization [14]. AAT belongs to the serine-proteinase-inhibitors, also known as Serpines that share the function but the tridimensional characteristics as well. Indeed, their structure is particularly prone to 
polymerization when a mutation occurs. These polymers tend to aggregate inside the cells, causing direct damage to the cell structure and acting as a neutrophilic chemotactic factor [15].

AAT behaves similarly. In particular, the $\mathrm{Z}$ mutation (Glu-toLys substitution at position 342), which is one of the most severe pathological variants [16], predisposes AAT to polymerization [17]. It is widely recognized that Z-AAT polymers gather inside the hepatocytes, particularly in the endoplasmic reticulum, where they directly damage the cell [18]. However, these polymers tend to gather also in the lungs, where they activate the neutrophils by inducing chemotaxis and stimulating the secretion of myeloperoxidase both in the alveolar and bronchial cells [19].

Along with the lack of effective anti-protease activity, this peculiar inflammatory damage to the lung parenchyma may contribute to the classic development of panlobular emphysema and subsequent chronic obstructive pulmonary disorder (COPD).

However, radiologic pictures of COPD sustained by centrilobular emphysema have also been described in Z-related AATD, where the chronic obstructive ventilatory defect is mainly the result of extensive small airway disease (SAD) [20]. SAD is caused by the progressive obstruction and disappearance of peripheral airways due to mucus endoluminal hypersecretion, submucosal accumulation of inflammatory cells, and smooth muscle cells hyperplasia-hypertrophy combined with the deposition of periadventitial fibrous tissue, leading to narrowing and early closure of these peripheral airways that become the main location of increased airflow resistance in COPD [21-26]. The unremitting inflammatory process sustained excessive oxidative stress, and protease- antiprotease imbalance is believed to be the main pathogenetic mechanisms of such disease, defined as fibrosing chronic bronchiolitis. Over time these mechanisms and related structural damages may peripherally involve the respiratory bronchioli, also affecting their alveolar attachments with the progressive development of centri-lobular emphysema [20,27-29].

It can be postulated that the occurrence of centri-lobular emphysema in PiZZ subjects affected by AATD might be the consequence of chronic bronchiolitis due to the pro-inflammatory activity of the polymers gathered in the bronchiolar epithelial cells that functionally can be reflected by the presence of SAD.

The existence of AAT polymers has been previously demonstrated in bronchial biopsies of subjects with Z-AAT deficiency $[30,31]$, but no studies have investigated their presence in the airways in a non-invasive way. Exhaled Breath Condensate (EBC) analysis could be a new tool to assess the presence of Z-AAT polymers in both large and small airways, reflecting the composition of the airway-lining fluid.

Our study aims to evaluate the function of the small airway and the presence of Z-AAT polymers in the EBC from subjects with PiZZ related AATD without COPD and comparing the results with similar tests performed on healthy PiMM subjects.

\section{Materials and Methods}

We enrolled the last 9 PiZZ subjects (cases) registered from the database of the Regional Referral Center for AATD of the Spedali Civili of Brescia (Italy) [32,33], and 10 PiMM healthy subjects (controls). All subjects included were of legal age, no smoking history, and normal baseline spirometry. A complete medical history was collected, and AATD was confirmed by AAT serum levels and by genotyping.
All subjects underwent complete pulmonary function tests (PFTs) (including spirometry to obtain directly measurable lung volumes and maximal flows), multi-breaths helium dilution technique in closed circuit for measurement of lung volumes (functional residual capacity: FRC; residual volume: RV; and total lung capacity: TLC), and determination of lung diffusing capacity for carbon monoxide (DLCO), obtained by measurement of alveolar volume (VA) and coefficient of transfer factor for $\mathrm{CO}$ (KCO) (BIOMEDIN Instruments, Padua, Italy). Multi-breath nitrogen wash-out test and single breath nitrogen wash-out test were also performed for determination of lung clearance index (LCI) and closing volume (CV), closing capacity (CC), and phase III N2-slope, respectively (Exhalizer D, Eco-Medics, Dürnten, Switzerland). EBC was obtained from both PiMM and PiZZ subjects by breathing throughout frozen R-Tubes. Collected EBC, added with a protease inhibitor cocktail (Sigma-Aldrich, St. Louis, MO, USA), and 0.1\% BSA were centrifuged, aliquoted and lyophilized. Lyophilized samples were resuspended in $1 / 10^{\text {th }}$ of the original volume. Concentrated $\mathrm{EBC}$ from $\mathrm{ZZ}$ or $\mathrm{MM}$ patients were subjected to SDS-PAGE and blotted as described [31]. Membranes were probed with anti-AAT antibodies (Dako Cytomation, Glostrup, Denmark) following manufactures' instructions and revealed with HRP-conjugated secondary antibodies and ECL method (GE-Health Care, Chicago, IL, USA). Commercially available purified hAAT (Gentaur, Bergamo, Italy) was loaded as a positive control. ELISA for total AAT was performed as previously described [31] with minor modifications. Briefly, 10x concentrated exhalates were added to the well of a 96-well plate (Corning Inc., Conring, NY, USA) previously coated with a polyclonal rabbit anti-human AAT antibody (Dako Cytomation). Purified hAAT was used for the standard curve. Next, HRP-conjugated polyclonal goat anti-human AAT antibody (Dako Cytomation) was added and, after the addition of TMB substrate (1-StepTM Ultra TMB-Elisa, Thermo Scientific), the absorbance of the developed color was estimated using a plate reader. ELISA for polymeric AAT was performed essentially as described with minor modification [31]. Briefly, Costar plates coated as described above were incubated with $10 \mathrm{x}$ concentrated EBC. Then, wells were incubated with ATZ11 antibody, an antibody specific for Z-AAT polymers, and incubated with a biotinconjugated anti-mouse antibody (GE-Health-Care) followed by streptavidin-conjugated HRP. After the addition of TMB substrate (1-StepTM Ultra TMB-ELISA, Thermo Fisher Scientific, Waltham, MA, USA), the optical density was measured using a plate reader.

All LFTs were performed in the Laboratory of Respiratory Physiopathology of the University of Brescia - Spedali Civili of Brescia, whereas the analysis of the EBC was performed at the Department of Molecular and Translational Medicine, University of Brescia, Italy.

The study was performed in accordance with the Helsinki declaration and was approved by the University Ethic Committee at the Department of Experimental and Clinical Science. All participants signed written informed consent upon enrolling.

\section{Statistics}

Continuous variables were compared using the Student's $t$-test for unpaired data. Data were expressed as mean \pm standard deviation, and categorical variables were recorded as frequencies and percentages. Statistical significance was taken as $\mathrm{p}<0.05$. 


\section{Results}

The anthropometric characteristics of the subjects (cases and controls) are shown in Table 1 . The mean age was $38.38 \pm 11.3$ years for the PiMM group, whereas the mean age for the PiZZ group was $38.1 \pm 8.0$ years. Similarly, there were no statistically significant differences between the two groups regarding weight, height, and body mass index. Per protocol, simple spirometry had to be normal; thus, no significant differences were expected in $\mathrm{VC}$, $\mathrm{FEV}_{1}$, and $\mathrm{FEV}_{1} / \mathrm{VC} \%$ ratio. Lung volumes were not different, and also VA, Kco, and DLCO were similar between the two groups (Table 1). The single-breath nitrogen test revealed a statistically significant difference between the two groups regarding the phase III N2-slope slope $(1.45 \pm 0.35$ vs $0.96 \pm 0.40 \mathrm{~N} 2 / \mathrm{L})(\mathrm{p}<0.02)$, while the closing volume/vital capacity $(\mathrm{CV} / \mathrm{VC}) \%$ ratio was not significantly increased $(14.3 \pm 4.5$ vs $11.3 \pm 6.2 \%)$ in PiZZ subjects. Although LCI was worse in the PiZZ subjects, it was not significantly different as compared to PiMM healthy subjects $(7.9 \pm 2.0 \mathrm{vs}$ 7.4 \pm 0.9 FRC turnover) (Table 2). Analysis of AAT by SDS-PAGE followed by western blot showed that AAT is present in concentrated EBC from both PiMM and PiZZ subjects (Figure 1A).

The quantification of total AAT in EBC by ELISA shows significant variability in AAT concentration among the subjects in both groups and that no statistically significant difference in total AAT concentration was present between PiMM and PiZZ patients (Figure 1B). In EBC from PiMM patients, polymeric AAT was not present, as expected. Polymeric AAT was instead detected in PiZZ subjects, but only in $44 \%$ of them (Figure 1C). The different levels of total AAT in EBC of PiZZ subjects prompted us to analyze if a higher total AAT concentration in EBC could lead to an increased polymeric AAT level. However, no significant correlation between total and polymeric AAT amount was found in the analyzed patients.
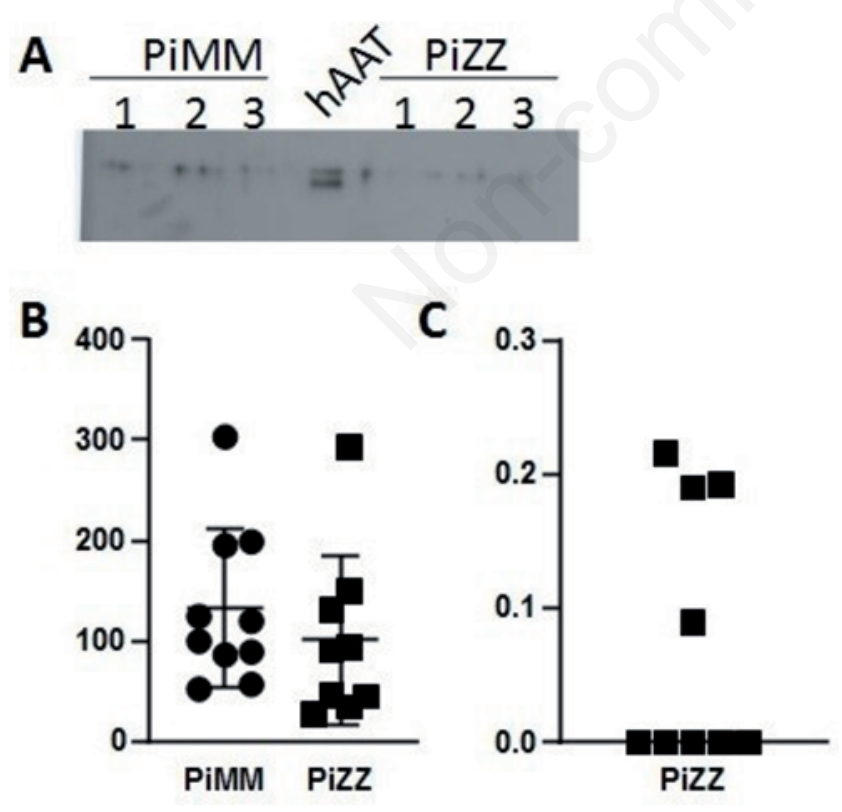

Figure 1. Western blot analysis using AAT antibody in 10-fold concentrated EBC from 3 PiMM and 3 PiZZ patients. Purified human serum AAT (h-AAT) served as a positive control. A typical fluorogram from three independent experiments is shown (A), which gave superimposable results. ELISA for total (B) and polymeric (C) AAT in 10-fold concentrated EBC.
Table 1. Anthropometric and functional respiratory data of controls and cases.

\begin{tabular}{|c|c|c|c|}
\hline Cont & trols (MM Sub) & Cases (ZZ Sub) & p-valu \\
\hline Age (years) & $38.8 \pm 11.3$ & $38.1 \pm 8.0$ & ns \\
\hline Sex (male/female) & $6 / 4$ & $3 / 6$ & ns \\
\hline Weight (Kg) & $70.6 \pm 18.4$ & $67.6 \pm 21.6$ & ns \\
\hline Height (m) & $1.69 \pm 11$ & $1.69 \pm 8$ & ns \\
\hline BMI $\left(\mathrm{Kg} / \mathrm{m}^{2}\right)$ & $23.3 \pm 1.7$ & $23.2 \pm 1.8$ & ns \\
\hline VC (L) & $4.73 \pm 1.12$ & $4.46 \pm 1.11$ & ns \\
\hline VC (\% pred.) & $111 \pm 11$ & $111 \pm 15$ & ns \\
\hline IC (L) & $3.18 \pm 0.80$ & $2.90 \pm 0.77$ & ns \\
\hline FVC (L) & $4.61 \pm 1.04$ & $4.36 \pm 1.09$ & ns \\
\hline FVC (\% pred) & $112 \pm 10$ & $111 \pm 15$ & ns \\
\hline $\mathrm{FEV}_{1}(\mathrm{~L})$ & $3.86 \pm 0.69$ & $3.57 \pm 0.89$ & ns \\
\hline $\mathrm{FEV}_{1}$ (\% pred.) & $113 \pm 9$ & $107 \pm 13$ & ns \\
\hline $\mathrm{FEV}_{1} \mathrm{NC}(\%)$ & $82.90 \pm 7.57$ & $80.25 \pm 7.66$ & ns \\
\hline $\mathrm{FEV}_{1}$ NC (\% pred.) & $102 \pm 7$ & $97 \pm 6$ & ns \\
\hline PEF (L/s) & $9.76 \pm 2.02$ & $8.34 \pm 2.28$ & ns \\
\hline PEF (\% pred.) & $120 \pm 15$ & $108 \pm 16$ & ns \\
\hline FEF25-75 (L/s) & $4.27 \pm 0.66$ & $3.77 \pm 1.38$ & ns \\
\hline FEF25-75 (\% pred.) & $106 \pm 19$ & $93 \pm 27$ & ns \\
\hline RV (L) & $1.71 \pm 0.47$ & $1.61 \pm 0.42$ & ns \\
\hline RV (\% pred.) & $98 \pm 26$ & $95 \pm 16$ & ns \\
\hline FRC (L) & $3.03 \pm 0.92$ & $3.08 \pm 0.78$ & ns \\
\hline FRC (\% pred.) & $97 \pm 23$ & $103 \pm 22$ & ns \\
\hline TLC (L) & $6.43 \pm 1.51$ & $6.07 \pm 1.45$ & ns \\
\hline TLC (\% pred.) & $106 \pm 9$ & $105 \pm 13$ & ns \\
\hline RV/TLC \% & $26.46 \pm 4.07$ & $26.70 \pm 3.15$ & ns \\
\hline RV/TLC (\% pred.) & $91 \pm 19$ & $87 \pm 9$ & ns \\
\hline DLco $(\mathrm{mL} \times \min \times \mathrm{mmHg})$ & $27.0 \pm 4.1$ & $23.8 \pm 5.9$ & ns \\
\hline DLco (\% pred.) & $92 \pm 11$ & $82 \pm 12$ & ns \\
\hline $\mathrm{Va}(\mathrm{L})$ & $6.08 \pm 1.39$ & $5.75 \pm 1.35$ & ns \\
\hline Va (\% pred.) & $103 \pm 8$ & $102 \pm 12$ & ns \\
\hline $\mathrm{Kco}(\mathrm{mL} \times \min \times \mathrm{mmHg}) / \mathrm{L}$ & $4.6 \pm 0.9$ & $4.2 \pm 0.90$ & ns \\
\hline Kco (\% pred.) & $90 \pm 12$ & $82 \pm 17$ & ns \\
\hline
\end{tabular}

BMI, body mass index; VC, vital capacity; \% pred., percentage of predicted value); IC, inspiratory capaci ty; ERV, expiratory reserve volume; FVC, forced vital capacity; $\mathrm{FEV}_{1}$, forced expiratory volume in the first second; PEF, peak expiratory flow; FEF25-75, forced expiratory flow at 25\%-75\%; RV, residual volume; FRC, functional residual capacity; TLC, total lung capacity; Va, alveolar volume; DLCO, carbonmonoxide diffusing capacity; KCO, Krogh's index; ns, not significance.

Table 2. Parameters related to ventilation inhomogeneity in controls and cases.

\begin{tabular}{lccc} 
& Controls (MM Sulb) & Cases (ZM Sulb) & p-value \\
CV (L) & $0.55 \pm 0.36$ & $0.60 \pm 0.21$ & $n s$ \\
CC (L) & $2.23 \pm 0.57$ & $2.36 \pm 0.37$ & $n s$ \\
\hline Phase III slope (N2\%/L) & $0.96 \pm 0.40$ & $1.45 \pm 0.35$ & $<0.02$ \\
CVNC \% & $11.30 \pm 6.23$ & $14.26 \pm 4.51$ & $\mathrm{~ns}$ \\
\hline LCI (FRC turnovers) & $7.4 \pm 0.9$ & $7.9 \pm 2.0$ & $\mathrm{~ns}$ \\
\hline
\end{tabular}

$\mathrm{CV}$, closing volume; $\mathrm{CC}$, closing capacity; $\mathrm{VC}$, vital capacity; LCI, lung clearance index. 


\section{Discussion}

The findings of this study demonstrated that in asymptomatic, never smoker PiZZ subjects who have normal spirometry and lung diffusion capacity, pulmonary function test such as single breath N2 wash-out, able to explore the degree of ventilation heterogeneity, as an indirect index of small airways involvement, showed parameters significantly different from those seen in matched healthy PiMM subjects. This strongly suggests that Z-AAT polymers, found in EBC in almost $50 \%$ of our PiZZ subjects, might promote a sustained inflammation in the bronchioli, possibly leading to COPD. Surprisingly, although PiZZ subjects showed a worse lung clearance index (LCI) than PiMM healthy subjects, suggesting a less effective alveolar air exchange, the difference was not statistically significant. If it is a problem of sample size or the method's sensitivity, this has to be elucidated in the future.

Previous literature data reported that AAT is present in EBC and that its concentration is too low to be detected in non-concentrated samples [34,35]. Accordingly, we detected AAT in concentrated EBC from PiMM, but also from PiZZ subjects, at a concentration that, even if quite variable among the different subjects, is comparable to what previously described. Moreover, we report the presence of AAT polymers in concentrated EBC in a subgroup of PiZZ patients ( 4 over 9, 44\%). The lack of a correlation between total AAT concentration and polymer detection suggests that conditions other than AAT concentration at the bronchial level may favor Z-AAT polymerization. In agreement with our group's previous results, Z-AAT polymers found in EBC are likely secreted or released by bronchial epithelial cells [31]. Indeed, we showed that, in bronchial biopsy of PiZZ patients, Z-AAT polymers are present in the bronchial epithelium and that Z-AAT polymers can be detected in culture media of ex-vivo cultured bronchial epithelial cells from PiZZ patients [31]. The finding that AAT polymers are unrelated to the total AAT concentration found in $\mathrm{EBC}$ also allowed to rule out the possibility that detected polymers are artifacts due to sample treatment. Since Z-AAT polymers' significant values in $\mathrm{EBC}$ were observed only in $4 \mathrm{PiZZ}$ subjects, correlations were not possible with PFTs. More studies are needed in order to analyze this stimulating aspect.

\section{Conclusions}

In this proof-of-concept study, asymptomatic, never smoker PiZZ subjects with normal spirometry and DLCO, in almost 50\% of whom Z-AAT polymers could be found in $\mathrm{EBC}$, showed greater ventilation heterogeneity, as documented by the increase of phase III slope during single N2 wash- out test, as compared to matched healthy PiMM subjects, suggesting a peripheral airway involvement. These findings suggest that the presence of Z-AAT polymers in the bronchial tree might induce pathological changes in the small airways through their pro-inflammatory activity, potentially contributing to the development of COPD.

\section{References}

1. Blanco I, Bueno P, Diego I, et al. Alpha-1 anti-trypsin Pi?Z gene frequency and Pi?ZZ genotype numbers worldwide: an update. Int J Chron Obstruct Pulmon Dis 2017;12:561-69.
2. Strnad P, McElvaney NG, Lomas DA. Alpha1-antitrypsin deficiency. N Engl J Med 2020;382:1443-55.

3. Franciosi AN, Carroll TP, McElvaney NG.Franciosi AN, et al. Pitfalls and caveats in alpha1- antitrypsin deficiency testing: a guide for clinicians. Lancet Respir Med 2019;7:1059-67.

4. Ferrarotti I, Ottaviani S, De Silvestri A, Corsico AG. Update on alpha(1)-antitrypsin deficiency. Breathe (Sheff) 2018; 14:e17-e24.

5. Janciauskiene S, Welte T. Well known and less well known functions of alpha-1 anti-trypsin: its role in chronic obstructive pulmonary disease and other disease developments. Ann Am Thorac Soc 2016;13:S280-8.

6. Greene CM, Marciniak SJ, Teckman J, et al. $\alpha 1$-antitrypsin deficiency. Nat Rev Dis Primers 2016;2:16051.

7. Stoller JK, Aboussouan LS. A review of $\alpha 1$-antitrypsin deficiency. AM J Respir Crit Care Med 2011;185:246-59.

8. Eriksson S, Carlson J, Velez R. Risk of cirrhosis and primary liver cancer in alpha1-antitrypsin deficiency. N Engl J Med 1986;314:736-9.

9. Hussain M, Mieli-Vergani G, Mowat AP. Alpha1-antitrypsin deficiency and liver disease: clinical presentation, diagnosis and treatment. J Inherit Metab Dis 1991;14:497-511.

10. Pini L, Paoletti G, Heffler E, et al. Alpha1- antitrypsin deficiency and asthma. Curr Opin Allergy Clin Immunol 2021;21:46-51.

11. Vizzardi E, Corda L, Sciatti E, et al. Echocardiographic evaluation in subjects with $\alpha 1$-antitrypsin deficiency. Eur J Clin Invest 2015;45:949-54.

12. Vizzardi E, Corda L, Pezzali N, et al. Elastic properties of the ascending aorta in patients with ?1-antitrypsin deficiency ( $\mathrm{Z}$ homozygotes). Heart 2012;98:1354-8.

13. Pini L, Peroni M, Zanotti $\mathrm{C}$, et al. Investigating the link between Alpha-1 antitrypsin deficiency and abdominal aortic aneurysms. Ann Vasc Surg 2021;77:195-201.

14. Lomas DA, Parfrey H. $\alpha 1$-Antitrypsin deficiency: Molecular pathophysiology. Thorax 2004;59:529-35.

15. Gooptu B, Lomas DA. Polymers and inflammation: disease mechanisms of the serpinopathies; J Exp Med 2008;205:1529-34.

16. Luisetti M, Seersholm N. $\alpha 1$-Antitrypsin deficiency: Epidemiology of $\alpha 1$-antitrypsin deficiency. Thorax 2004; 59164-9.

17. Lomas DA, Mahadeva R. Alpha1-antitrypsin polymerization and the serpinopathies: pathobiology and prospects for therapy. J Clin Invest 2002;110:1585-90.

18. Lomas DA, Evans DL, Finch JT, Carrell RW. The mechanism of $\mathrm{Z}$ alpha1-antitrypsin accumulation in the liver. Nature 1992;357:605-7.

19. Parmar JS, Mahadeva R, Reed BJ, et al. Polymers of alpha-1 anti-trypsin are chemotactic for human neutrophils: a new paradigm for the pathogenesis of emphysema. Am J Respir Cell Mol Biol 2002;26:723-30.

20. Koo HK, Vasilescu DM, Booth S, et al. Small airways disease in mild and moderate chronic obstructive pulmonary disease: a cross-sectional study. Lancet Respir Med 2018;6:591-602.

21. Yanai M, Sekizawa K, Ohrui T, et al. Site of airway obstruction in pulmonary disease: direct measurement of intrabronchial pressure. J Appl Physiol 1992;72:1016-23.

22. Kim, Rogers TJ, Criner GJ. New concepts in the pathobiology of chronic obstructive pulmonary disease. Proc Am Thorac Soc 2008;5:478.

23. Taraseviciene-Stewart L, Voekel NF. Molecular pathogenesis of emphysema. J Clin Invest 2008;118:394.

24. Hogg JC, Timens W. The pathology of chronic obstructive pul- 
monary disease. Ann Rev Path Mech Dis 2009;4:435-59.

25. Tantucci C, Bottone D, Levi G, et al. Respiratory function, autonomic dysfunction, and systemic inflammation are closely linked in patients with COPD and tidal flow limitation: An exploratory study. Respir Physiol Neurobiol 2021;284:103565.

26. Uccelli S, Pini L, Bottone D, et al. Dyspnea during night-time and at early morning in patients with stable COPD is associated with supine tidal expiratory flow limitation. Int J Chron Obstruct Pulmon Dis 2020;15:2549-58.

27. Burgel PR. The role of small airways in obstructive airway diseases. Eur Respir Rev 2011;20:23-33.

28. Stockley JA, Cooper BG, Stockley RA, Sapey E. Small airways disease: time for a revisit? Int J Chron Obstruct Pulmon Dis $2017 ; 12: 2343-53$

29. Tantucci C, Pini L. Inhaled corticosteroids in COPD: Trying to make a long story short. Int J Chron Obstruct Pulmon Dis 2020;15:821-9.
30. Bazzan E, Tinè M, Biondini D, et al. Alpha1-Antitrypsin polymerizes in alveolar macrophages of smokers with and without alpha1-antitrypsin deficiency. Chest 2018;154:607-16.

31. Pini L, Tiberio L, Venkatesan N, et al. The role of bronchial epithelial cells in the pathogenesis of COPD in Z-alpha-1 antitrypsin deficiency. Respir Res 2014;15:112.

32. Corda L, Bertella E, Pini L, et al. Diagnostic flow chart for targeted detection of alpha1- antitrypsin deficiency. Respir Med 2006;100:463-70.

33. Pini L, Corda L, Malerba M, et al. Alpha 1-antitrypsin deficiency: the Brescia clinical study. Recenti Prog Med 2000;91:352-61.

34. Bayley DL, Abusriwil H, Ahmad A, Stockley RA. Validation of assays for inflammatory mediators in exhaled breath condensate. Eur Respir J 2008;31:943-8.

35. Koczulla AR, Noeske S, Herr C, et al. Alpha-1 anti-trypsin is elevated in exhaled breath condensate and serum in exacerbated COPD patients. Respir Med 2012;106:120-6. 